\title{
Live four-dimensional optical coherence tomography reveals embryonic cardiac phenotype in mouse mutant
}

Andrew L. Lopez, III

Shang Wang

Kirill V. Larin

Paul A. Overbeek

Irina V. Larina

\section{SPIE.}




\section{Live four-dimensional optical coherence tomography reveals embryonic cardiac phenotype in mouse mutant}

\author{
Andrew L. Lopez III, ${ }^{a}$ Shang Wang, ${ }^{a}$ Kirill V. Larin, ${ }^{\text {a,b,c }}$ \\ Paul A. Overbeek, ${ }^{d}$ and Irina V. Larina ${ }^{a, *}$ \\ aBaylor College of Medicine, Department of Molecular Physiology and \\ Biophysics, One Baylor Plaza, Houston 77030, United States \\ bUniversity of Houston, Department of Biomedical Engineering, 3605 \\ Cullen Boulevard, Houston 77204, United States \\ 'Samara State Aerospace University, 34 Moskovskoye Shosse, \\ Samara 443086, Russia \\ ${ }^{d}$ Baylor College of Medicine, Department of Molecular \& Cellular \\ Biology, One Baylor Plaza, Houston 77030, United States
}

\begin{abstract}
Efficient phenotyping of developmental defects in model organisms is critical for understanding the genetic specification of normal development and congenital abnormalities in humans. We previously reported that optical coherence tomography (OCT) combined with live embryo culture is a valuable tool for mouse embryo imaging and four-dimensional (4-D) cardiodynamic analysis; however, its capability for analysis of mouse mutants with cardiac phenotypes has not been previously explored. Here, we report 4-D (three-dimensional+time) OCT imaging and analysis of the embryonic heart in a Wdr19 mouse mutant, revealing a heart looping defect. Quantitative analysis of cardiac looping revealed a statistically significant difference between mutant and control embryos. Our results indicate that live 4-D OCT imaging provides a powerful phenotyping approach to characterize embryonic cardiac function in mouse models. () 2015 Society of Photo-Optical Instrumentation Engineers (SPIE) [DOI: 10.1117/1.JBO.20.9.090501]
\end{abstract}

Keywords: optical coherence tomography; cardiovascular development; mouse; embryology; cardiodynamics.

Paper 150463LR received Jul. 8, 2015; accepted for publication Aug. 14, 2015; published online Sep. 21, 2015.

Congenital heart defects are the most common birth defects. Our understanding of normal and abnormal cardiovascular development has been aided by phenotypic analysis of mutant mouse phenotypes. ${ }^{1}$ Traditional imaging techniques, such as high-frequency ultrasonic imaging ${ }^{2}$ and confocal fluorescence imaging, ${ }^{3}$ have been used for live phenotyping of the cardiovascular system in mouse embryos. However, these methods suffer from either low spatial resolution or limited imaging depth. Optical coherence tomography (OCT) is a depth-resolved three-dimensional (3-D) imaging modality with the spatial scales that fill the gap among the traditional imaging methods. ${ }^{4}$ Previously, our group has combined OCT with mouse embryo culture for live imaging of cardiodynamics and hemodynamics in mouse embryos. ${ }^{5-7}$ However, the feasibility of applying OCT to capture and characterize mutant cardiac phenotypes in mouse embryos was not investigated before. Here, we present the first study of using four-dimensional (4-D) (3-D+time) OCT for dynamic imaging of early embryonic heart in the mouse embryos with a mutation in the Wdr19 gene. Our imaging studies reveal a dramatic cardiac looping defect. These results demonstrate that 4-D OCT is a powerful phenotyping approach for genetically engineered mouse lines with altered cardiac morphogenesis and function.

WDR19 (IFT144) is a member of the family of WD-repeat proteins. ${ }^{8}$ WDR19 mutations have been found in human patients with certain ciliopathies, including Sensenbrenner and Jeune syndromes. ${ }^{9}$ The Wdr19 mouse line used in this study was created using random insertional mutagenesis (data not shown). Heterozygous mice are viable and do not exhibit obvious defects; homozygous Wdr19 mutants are embryonic lethal at about embryonic day 10.5 (E10.5) exhibiting defects in neural tube closure and brain development.

Matings of heterozygous Wdr19 mice were set up overnight and checked daily for vaginal plugs. The day of the observed vaginal plug was counted as E0.5. Embryos were dissected at E8.5 with the yolk sac intact in a culture medium containing 89\% DMEM/F12, 1\% Pen-strep solution, and 10\% fetal bovine serum. The dissection station was maintained at $37^{\circ} \mathrm{C}$. After dissection, live embryos were transferred to a humidified incubator maintained at $37^{\circ} \mathrm{C}, 5 \% \mathrm{CO}_{2}$ and allowed to recover for at least $60 \mathrm{~min}$. The dish and the embryos were then transferred to the OCT imaging stage, maintained at $37^{\circ} \mathrm{C}, 5 \% \mathrm{CO}_{2}$ for data acquisition. Embryos at E9.5 were also dissected and imaged without the yolk sac attached for better visualization of the neural tube closure defect. After the imaging, embryos were genotyped by polymerase chain reaction. Wildtype and heterozygous embryos were grouped together and used as controls. All animal manipulation procedures described here were approved by the Animal Care and Use Committee of the Baylor College of Medicine and were carried out in accordance with the approved guidelines and regulations.

We utilized a home-built spectroscopic OCT system in this study. The details of the system were described in our previous work. ${ }^{10}$ Briefly, the OCT system employs a Ti:sapphire laser with a central wavelength of $\sim 808 \mathrm{~nm}$ and a bandwidth of $\sim 110 \mathrm{~nm}$. Interference fringes from a Michelson interferometer are detected by a high-speed spectrometer with a CMOS camera. The system has an A-line acquisition speed of up to $250 \mathrm{kHz}$. We obtained an axial resolution of $\sim 5 \mu \mathrm{m}$ in tissue with a transverse resolution of $\sim 4 \mu \mathrm{m}$. The sensitivity of the OCT system was measured to be $\sim 97 \mathrm{~dB}$. The system had an imaging depth of $\sim 5.5 \mathrm{~mm}$ in air and provided an available depth range of $\sim 1 \mathrm{~mm}$ for embryonic imaging due to the light attenuation in tissue.

The embryonic mouse heart beats at $\sim 2 \mathrm{~Hz}$ at the studied stages. Therefore, direct volumetric imaging with traditional OCT systems cannot provide a sufficient volume rate for cardiodynamic analysis. To overcome this limitation, we conducted B-scan imaging over time at different locations throughout the heart in parallel slice geometry with a step (at least two cardiac cycles at each position). The B-scan time lapses were synchronized to the same phase of the heartbeat cycle as previously 


\section{JBO Letters}

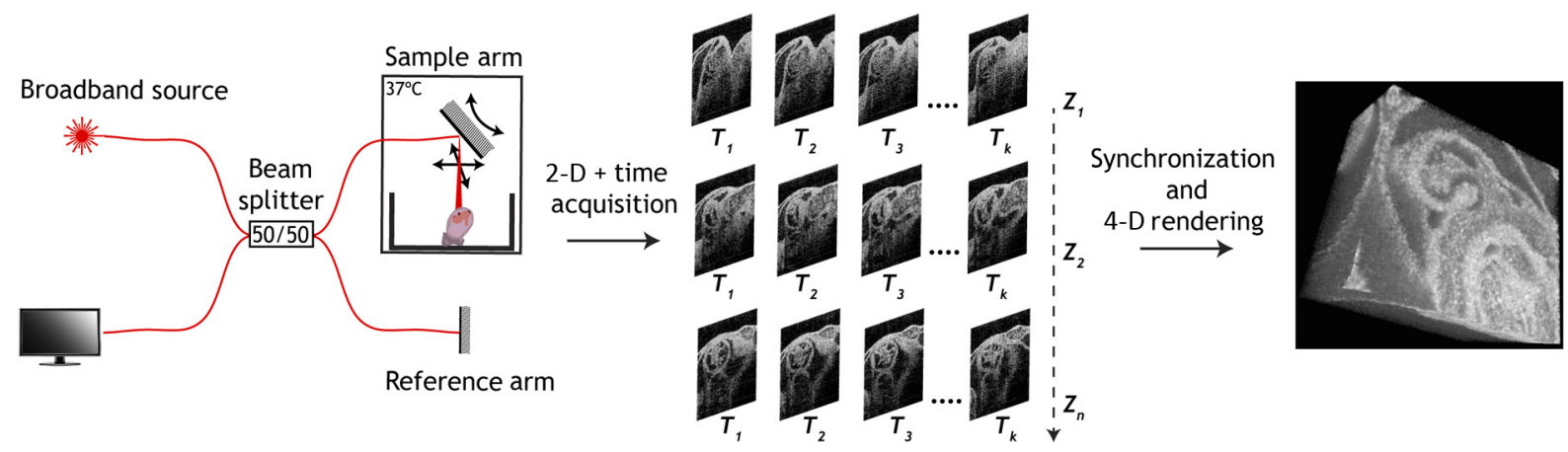

Fig. 1 Optical coherence tomography (OCT) data acquisition and synchronization in postprocessing for reconstruction of four-dimensional (4-D) cardiodynamics in live mouse embryos.

reported, ${ }^{11}$ which resulted in the same postprocessing volume rate as the OCT acquisition frame rate ${ }^{12-14}$ (Fig. 1). Time lapses were acquired at 600 A-lines per frame and a frame rate of $100 \mathrm{~Hz}$ ( $\sim 50$ volumes per heartbeat), providing a 4-D temporal resolution of $10 \mathrm{~ms}$. The acquisition time for each 4-D data set is $5 \mathrm{~min}$. The reconstruction algorithm is based on the assumptions that all cardiac cycles are identical within the acquisition time and that during this time the embryo and specifically the heart does not go through developmental changes. The Imaris software (Bitplane, Switzerland) and MATLAB ${ }^{\circledR}$ (MathWorks, Massachusetts) were used for data rendering, synchronization, 4-D visualization, and analysis. The characterization of the heart looping was accomplished by measuring the angle of the heart tube formed with respect to the inflow vessels where the blood is fed into the cardiac system. To do this, the OCT structural volume of the heart was first positioned with the ventral side en face; a clipping plane was used to acquire a cross-section through the sinus venosus, the primitive atrium, and the primitive ventricle. The reference angular position was determined as the bisector of the angle formed by the two inflow vessels.
The heart tube was represented by the line going through the center of the primitive atrium and the center of the primitive ventricle. The looping angle was measured from the reference angular position to the heart tube line.

Figure 2 shows representative 3-D OCT images of E9.5 and E8.5 control and Wdr19 mutant embryos together with optical microscopic images. It can be clearly seen that at the stage of E9.5 [Figs. 2(a) and 2(c)], the control embryo has a well-closed neural tube in the head region; in comparison, the neural tube remains open in the Wdr19 mutant embryo. Similar observations are also clear in E8.5 embryos, as shown in Figs. 2(b) and 2(d). In addition, at E8.5, the 3-D OCT images [Figs. 2(b) and 2(d)] clearly show a difference in the morphology of the heart tube between the control and the mutant embryos, suggesting a cardiac looping defect. The mouse embryonic heart starts to beat at E8.5, and our next step was to visualize early embryonic cardiodynamics at this early circulation stage to understand if the primary cardiac defect is functional or structural.

Figure 3 and Videos 1-3 show the 4-D cardiodynamics in E8.5 control and Wdr19 mutant embryos. The motion of the
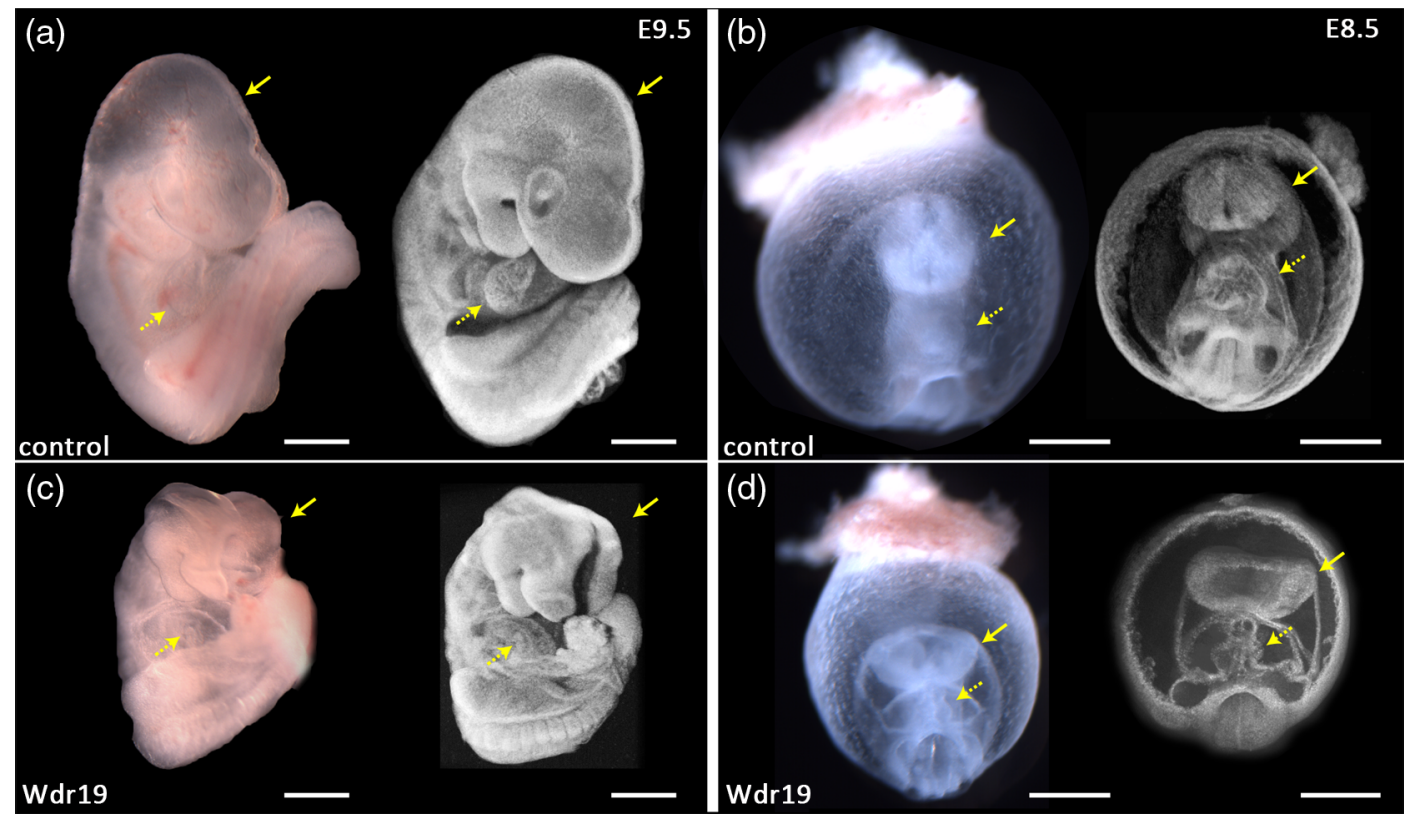

Fig. 2 Structural analysis of Wdr19 mutants. Optical microscopic images (left) and three-dimensional OCT images (right) of control [(a) and (b)] and Wdr19 mutant [(c) and (d)] mouse embryos at E9.5 [(a) and (c)] and E8.5 [(b) and (d)]. Solid arrows point at the neural tube in the head region and dashed arrows point at the heart. Scale bars correspond to $500 \mu \mathrm{m}$. 

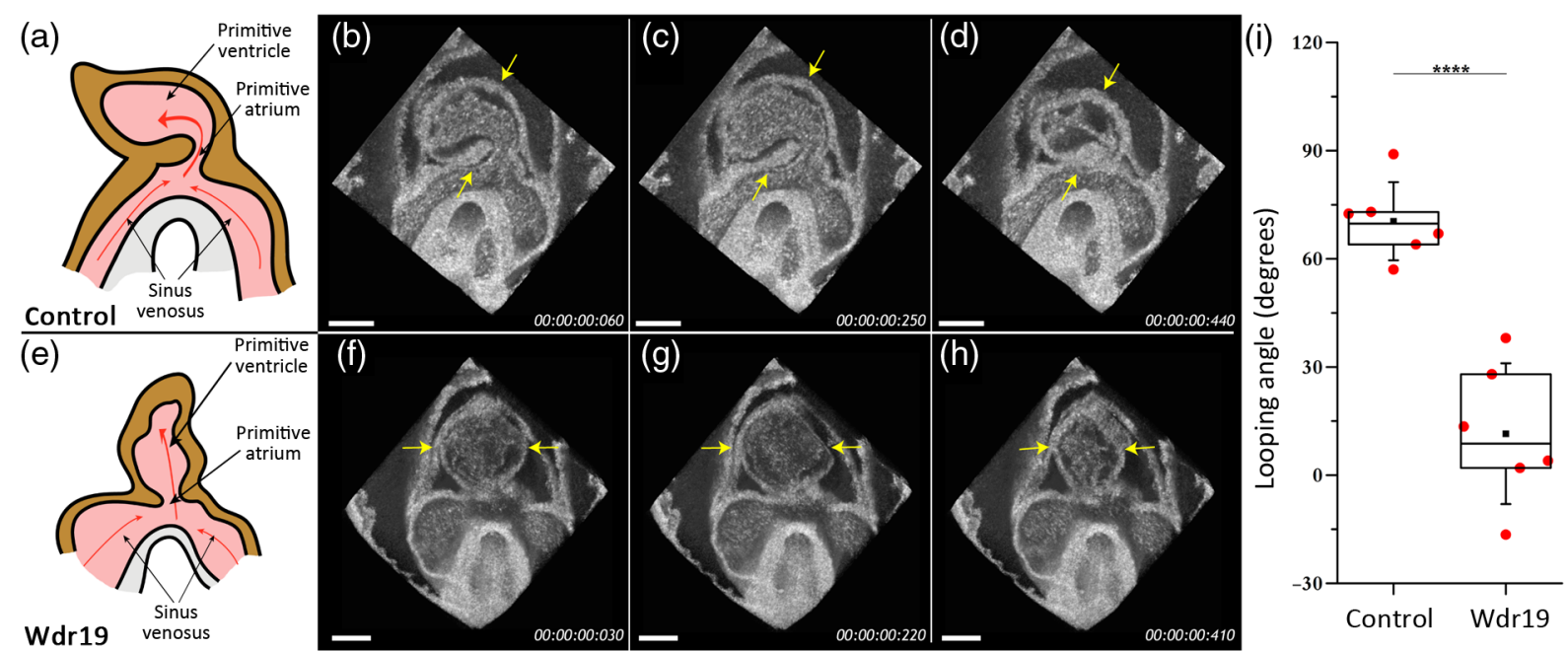

Fig. 3 Cardiodynamic analysis in E8.5 Wdr19 embryos. Illustrations represent cross-sections through 4-D OCT volumes at different phases of the heartbeat in control [(a)-(d)] and Wdr19 mutant [(e)-(h)] (Video 1, MOV, 10.3 MB [URL: http://dx.doi.org/10.1117/1.JBO.20.9.090501.1] and Video 2 MOV, 10.2 MB [URL: http://dx.doi.org/10.1117/1.JBO.20.9.090501.2], respectively). Video 3 (MOV, 6.97 MB [URL: http://dx.doi.org/10.1117/1.JBO.20.9.090501.3]) shows side-by-side comparison of cardiodynamics in wildtype and Wdr19 mutant embryos. (i) Box plot characterization of cardiac looping in Wdr19 mutants. Red dots show the measured values, solid squares represent the mean, and whiskers correspond to the standard deviation. The number of analyzed embryos $N=6$ for both control and mutant groups. ${ }^{* * *} p<0.0001$ from a two-sample two-tailed Student's $t$ test. The scale bars correspond to $200 \mu \mathrm{m}$.

heart wall and strong blood circulation are clearly seen, and the heart rate of the mutant embryos is in the normal range; however, there is an obvious heart tube looping defect. This structural defect is illustrated in Figs. 3(a) and 3(e). The quantification of the cardiac looping from the 3-D OCT data sets revealed a significantly smaller looping angle in the Wdr19 mutant hearts compared with the control ones based on the $p$ value of $\sim 0.00007,<0.0001$ from a two-sample two-tailed Student's $t$ test. These results imply a role for Wdr19 in the establishment or manifestation of left-right asymmetry during proper heart development.

It is known that Wdr19 is a ciliary protein that is involved in the formation and maintenance of cilia. ${ }^{15}$ Since previous studies have reported that the embryonic nodal flow introduced by the rotation of cilia plays a critical role in establishment of left-right asymmetry, ${ }^{16}$ the looping defect in the developing heart associated with Wdr19 disruption revealed in our study fits well with previous data. Further studies integrating live embryonic cardiodynamic imaging with molecular genetic approaches in Wdr19 mutants will help to elucidate the underlying signaling defects.

Strong blood circulation and normal heart rate in Wdr19 embryos are observed at early stages. However, abnormal looping can have secondary effects on blood circulation and vascular remodeling during heart development. Potentially, our approach can be used to assess time-dependent parameters like blood flow or heart rate during development.

While this study only utilized structural OCT imaging, functional OCT methods, such as Doppler OCT, ${ }^{17}$ speckle variance OCT,${ }^{18-23}$ and OCT-based heart wall dynamic imaging, ${ }^{24}$ can potentially be implemented and provide additional information about vascular remodeling and cardiac function.

The OCT system utilized in this study has a central wavelength of $808 \mathrm{~nm}$ and provides an imaging depth of $\sim 1 \mathrm{~mm}$ in the embryo. At E8.5 stage, this allows visualization of the whole embryo within the yolk sac. By E9.5 stage, as the embryo grows and turns, imaging the heart is possible when properly oriented; however, the embryo proper is poorly visualized. Utilizing a longer wavelength of $1300 \mathrm{~nm}$ allows deeper imaging due to greater light penetration in tissue. ${ }^{25}$

In conclusion, we demonstrate the use of 4-D OCT cardiodynamic imaging combined with embryo culture for live phenotyping of an early embryonic defect in cardiac morphogenesis. Through dynamic visualization and quantitative analysis, we report the discovery of a cardiac looping defect in Wdr19 mutant mouse embryos. This study indicates that 4-D OCT imaging is a useful tool for cardiovascular structural and functional phenotyping of early-stage embryos from genetically engineered mouse lines.

\section{Acknowledgments}

This work is supported by the National Institutes of Health (R01HL120140, U54HG006348, and R25GM056929) and the Optical Imaging and Vital Microscopy Core at the Baylor College of Medicine.

\section{References}

1. A. Moon, "Mouse models of congenital cardiovascular disease," Curr. Top. Dev. Biol. 84, 171-248 (2008).

2. F. S. Foster, J. Hossack, and S. L. Adamson, "Micro-ultrasound for preclinical imaging," Interface Focus 1(4), 576-601 (2011).

3. A. L. Lopez, III et al., "Live confocal microscopy of the developing mouse embryonic yolk sac vasculature," Methods Mol. Biol. 1214, 163-172 (2015).

4. D. Huang et al., "Optical coherence tomography," Science 254, 1178-1181 (1991).

5. I. V. Larina et al., "Live imaging of blood flow in mammalian embryos using Doppler swept-source optical coherence tomography," J. Biomed. Opt. 13, 060506 (2008).

6. I. V. Larina et al., "Sequential turning acquisition and reconstruction (STAR) method for four-dimensional imaging of cyclically moving structures," Biomed. Opt. Express 3, 650-660 (2012). 


\section{JBO Letters}

7. M. Garcia et al., "Imaging of cardiovascular development in mammalian embryos using optical coherence tomography," in Vascular Morphogenesis, D. Ribatti, Ed., pp. 151-161, Springer, New York (2015).

8. T. F. Smith, "Diversity of WD-repeat proteins," Subcell. Biochem. 48, 20-30 (2008)

9. H. Fehrenbach et al., "Mutations in WDR19 encoding the intraflagellar transport component IFT144 cause a broad spectrum of ciliopathies," Pediatr. Nephrol. 29, 1451-1456 (2014).

10. S. H. Syed et al., "Optical coherence tomography guided microinjections in live mouse embryos: high-resolution targeted manipulation for mouse embryonic research," J. Biomed. Opt. 20, 051020 (2015).

11. M. Liebling et al., "Four-dimensional cardiac imaging in living embryos via postacquisition synchronization of nongated slice sequences," J. Biomed. Opt. 10, 054001 (2005).

12. K. V. Larin et al., "Live Imaging of early developmental processes in mammalian embryos with optical coherence tomography," J. Innov. Opt. Health Sci. 2, 253-259 (2009).

13. A. Liu et al., "Efficient postacquisition synchronization of 4-D nongated cardiac images obtained from optical coherence tomography: application to 4-D reconstruction of the chick embryonic heart," J. Biomed. Opt. 14, 044020 (2009).

14. M. Gargesha et al., "High temporal resolution OCT using image-based retrospective gating," Opt. Express 17, 10786-10799 (2009).

15. R. G. Coussa et al., "WDR19: an ancient, retrograde, intraflagellar ciliary protein is mutated in autosomal recessive retinitis pigmentosa and in Senior-Loken syndrome," Clin. Genet. 84, 150-159 (2013).
16. N. Hirokawa et al., "Nodal flow and the generation of left-right asymmetry," Cell 125, 33-45 (2006).

17. M. W. Jenkins et al., "Measuring hemodynamics in the developing heart tube with four-dimensional gated Doppler optical coherence tomography," J. Biomed. Opt. 15, 066022 (2010).

18. N. Sudheendran et al., "Speckle variance OCT imaging of the vasculature in live mammalian embryos," Laser Phys. Lett. 8, 247-252 (2011).

19. P. M. Kulkarni et al., "Algorithms for improved 3-D reconstruction of live mammalian embryo vasculature from optical coherence tomography data," Quant. Imaging Med. Surg. 5, 125-135 (2015).

20. A. Mariampillai et al., "Speckle variance detection of microvasculature using swept-source optical coherence tomography," Opt. Lett. 33, 15301532 (2008).

21. E. Jonathan, J. Enfield, and M. J. Leahy, "Correlation mapping method for generating microcirculation morphology from optical coherence tomography (OCT) intensity images," J. Biophotonics 4, 583-587 (2011).

22. T. Kamali et al., "Assessment of transcutaneous vaccine delivery by optical coherence tomography," Laser Phys. Lett. 9, 607 (2012).

23. A. Doronin and I. Meglinski, "Imaging of subcutaneous microcirculation vascular network by double correlation optical coherence tomography," Laser Photonics Rev. 7, 797-800 (2013).

24. X. Yin et al., "Extracting cardiac shapes and motion of the chick embryo heart outflow tract from four-dimensional optical coherence tomography images," J. Biomed. Opt. 17, 096005 (2012).

25. S. H. Syed et al., "Optical coherence tomography for high-resolution imaging of mouse development in utero," J. Biomed. Opt. 16, 046004 (2011). 